\title{
Need for Deprescribing in Hospital Elderly Patients Discharged with a Limited Life Expectancy: The REPOSI Study
}

\author{
Luca Pasina $^{a}$ Barbara Brignolo Ottolini ${ }^{b} \quad$ Laura Cortesi $^{a} \quad$ Mauro Tettamantia \\ Carlotta Franchi $^{a}$ Alessandra Marengonic Pier Mannuccio Mannuccid \\ Alessandro Nobilia the REPOSI Investigators

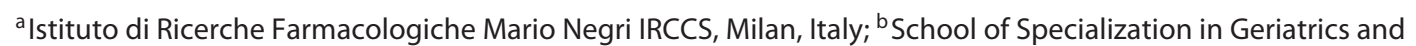 \\ Gerontology, University of Milan, Milan, Italy; ${ }^{\complement}$ Geriatric Unit, Spedali Civili, Department of Medical and Surgery \\ Sciences, University of Brescia, Brescia, Italy; ${ }^{d}$ IRCCS Ca' Granda Maggiore Hospital Foundation and University of \\ Milan, Milan, Italy
}

\section{Significance of the Study}

- Pharmacotherapy in older patients with a limited life expectancy should avoid unnecessary polypharmacy and focus on symptom control. This study showed a small reduction in the use of commonly prescribed preventive medications. Medications could be reviewed to optimize polypharmacy.

\section{Keywords}

Elderly . End of life - Limited life expectancy ·

Polypharmacy · Symptomatic medications

\begin{abstract}
Objective: Older people approaching the end of life are at a high risk for adverse drug reactions. Approaching the end of life should change the therapeutic aims, triggering a reduction in the number of drugs. The main aim of this study is to describe the preventive and symptomatic drug treatments prescribed to patients discharged with a limited life expectancy from internal medicine and geriatric wards. The secondary aim was to describe the potentially severe drug-drug interactions (DDI). Materials and Methods: We analyzed Registry of Polytherapies Societa Italiana di Medicina Interna (REPOSI), a network of internal medicine and geriatric wards,
\end{abstract}

to describe the drug therapy of patients discharged with a limited life expectancy. Results: The study sample comprised 55 patients discharged with a limited life expectancy. Patients with at least 1 preventive medication that could be considered for deprescription at the end of life were significantly fewer from admission to discharge ( $n=30 ; 54.5 \%$ vs. $n=21 ; 38.2 \% ; p=0.02$ ). Angiotensin-converting enzyme inhibitors, angiotensin II receptor blockers, calcium channel blockers, lipid-lowering drugs, and clonidine were the most frequent potentially avoidable medications prescribed at discharge, followed by xanthine oxidase inhibitors and drugs to prevent fractures. Thirty-seven (67.3\%) patients were also exposed to at least 1 potentially severe DDI at discharge. Conclusion: Hospital discharge is associated with a small reduction in the use of commonly prescribed preventive medications in patients discharged with a limited life expectancy. Cardiovascular drugs are the most frequent po-

\section{KARGER}

E-Mail karger@karger.com www.karger.com/mpp

\section{(C) 2019 The Author(s)}

Published by S. Karger AG, Basel

Karger

Open access

This is an Open Access article licensed under the Creative Commons Attribution-NonCommercial-4.0 International License (CC BY-NC) (http://www.karger.com/Services/OpenAccessLicense), applicable to the online version of the article only. Usage and distribution for commercial purposes requires written permission.
Luca Pasina

Pharmacotherapy and Appropriateness of Drug Prescription Unit

Istituto di Ricerche Farmacologiche Mario Negri IRCCS

Via Mario Negri 2, IT-20156 Milan (Italy)

E-Mail luca.pasina@marionegri.it 
tentially avoidable preventive medications. A consensus framework or shared criteria for potentially inappropriate medication in elderly patients with limited life expectancy could be useful to further improve drug prescription.

(c) 2019 The Author(s)

Published by S. Karger AG, Basel

\section{Introduction}

The end of life is the preterminal phase of a person's decline towards death and normally involves accumulating health problems over a period of weeks to months, with failing homeostasis that is irreversible and inexorably leads to death [1]. Older people approaching the end of life are at a high risk for adverse drug reactions resulting from polypharmacy, declining organ function, comorbidity, malnutrition, cachexia, and changes in body composition [2]. As a consequence, approaching the end of life should shift the therapeutic aims, triggering a reduction in the number of drugs, but this is not the usual practice [3-5].

The aim of pharmacotherapy in the rising numbers of end-of-life older people should be to avoid polypharmacy and focus on controlling symptoms rather than prolonging life $[1,2]$. Although polypharmacy is very common among older end-of-life patients, with their need to relieve symptoms, disease-related problems, and quality of life [6-8], it may also be a major risk factor for inappropriate prescribing and potentially severe drug-drug interactions (DDI) $[2,9,10]$. In this circumstance, drugs for the prevention of secondary diseases are of no value if the time to therapeutic benefit exceeds the probable life expectancy and should be discouraged. However, the few studies that have examined drug therapy in patients at the end of life have found that about half of them continued to take ineffective and unnecessary medication for the prevention or treatment of chronic diseases [11], although the potential for harm can be expected to outweigh any benefit in view of the limited life expectancy $[6$, 12]. Statins, antihypertensives and bisphosphonates are frequently used in patients with a terminal disease and advanced disability, although the real benefits are unknown because their safety and efficacy have been demonstrated for a young general population but evidence supporting their utility at the end of life is limited [1214].

No study has examined the prescription of internal and geriatric hospital specialists to patients with a limited life expectancy. The main aim of this study was to describe the preventive and symptomatic drug treatments prescribed to patients discharged with a limited life expectancy from internal medicine and geriatric wards. The secondary aim was to describe the potentially severe DDI.

\section{Materials and Methods}

\section{Data Collection}

The Registry of Polytherapies Societa Italiana di Medicina Interna (REPOSI) is a collaborative, independent initiative of the Italian Society of Internal Medicine (SIMI), the Istituto di Ricerche Farmacologiche Mario Negri IRCCS, and the IRCCS Ca' Granda Maggiore Policlinico Hospital Foundation. The registry was set up in 2008 by a network of internal medicine and geriatric wards in order to collect information on elderly hospital in-patients with multimorbidities receiving multiple drugs. The first run of data collection was between January and December 2008, and the second, third, and fourth runs as well as ongoing runs took place between January and December 2010, 2012, 2014, and 2016, respectively. To ensure an unselected population of elderly patients admitted to internal medicine and geriatric wards, the first 5 patients admitted to the wards participating in this study during 4-week periods 3 months apart were consecutively recruited if they were 65 years old or older. Participation was voluntary and all patients gave signed informed consent. Data collection complied fully with Italian law on personal data protection and this study was approved by the ethics committees of each ward participating in REPOSI.

The attending physicians completed a standardized web-based case report form, recording sociodemographic details and the diagnosis and drug treatment at admission, during the hospital stay, and at discharge. From the second REPOSI runs we collected the following additional information for a short-term follow-up in order to improve the quality of the data: main laboratory parameters, comorbidity according to the Cumulative Illness Rating Scale (CIRS), basic activities of daily living, cognitive impairment, depression, and clinical events during the hospital stay. Patients were followed for 3 months after discharge via a telephone interview in order to collect information on new diagnoses, hospital readmissions, drug regimens, adverse events, basic activities of daily living, and mortality.

To describe the drug therapy of patients discharged with a limited life expectancy, we considered eligible for analysis all patients discharged in "critical condition," defined as a high risk of shortterm mortality (at 3 months) on the basis of the clinical evaluation. Analysis of each patient's drug therapy at admission reflects drugs prescribed by general practitioners for patients who lived at home, or physicians in nursing homes for those in an institution; drug therapy at discharge referred to prescriptions by hospital internists or geriatricians. Patients transferred to palliative care wards, though potentially terminally ill, were excluded because the drug therapy at discharge was not collected.

\section{Classification of Drug Therapies}

The drugs prescribed at admission and discharge were divided into the following 3 main classes according to their preventive or symptomatic effects: (1) potentially avoidable preventive medications, i.e., drugs that usually have no place in the end-of-life patient because the time to benefit is clearly shorter than the life expec- 
Table 1. Main characteristics and diagnosis of the 55 patients discharged in critical condition

\begin{tabular}{|c|c|}
\hline Characteristic & Value \\
\hline Age, years & $80.8 \pm 83$ \\
\hline Female sex, \% & 43.6 \\
\hline Caregiver & $38(71.7)$ \\
\hline \multicolumn{2}{|c|}{ Hospital admission in the previous } \\
\hline 6 months & $27(49.1)$ \\
\hline BMI & $23.6 \pm 3.7$ \\
\hline Underweight & $4(9.7)$ \\
\hline Normal & $25(61.0)$ \\
\hline Overweight & $12(29.3)$ \\
\hline Bedridden & $20(40.8)$ \\
\hline Pressure sores & $24(66.7)$ \\
\hline Systolic blood pressure & $116.2 \pm 22.1$ \\
\hline Diastolic blood pressure & $67.1 \pm 12.4$ \\
\hline Short Blessed Test & $15.6 \pm 8.6$ \\
\hline Barthel index & $54.7 \pm 34.4$ \\
\hline Geriatric depression scale & $1.8 \pm 1.1$ \\
\hline \multicolumn{2}{|c|}{ Cumulative illness rating scale } \\
\hline Severity index & $1.9 \pm 0.4$ \\
\hline Comorbidity index & $3.9 \pm 2.1$ \\
\hline \multicolumn{2}{|l|}{ Main diagnosis } \\
\hline Hypertension & $41(74.6)$ \\
\hline Heart failure & $15(27.3)$ \\
\hline Chronic renal failure & $13(23.6)$ \\
\hline Atrial fibrillation & $14(25.4)$ \\
\hline Cancer & $23(41.8)$ \\
\hline Diabetes & $13(23.6)$ \\
\hline Ischemic heart disease & $14(25.4)$ \\
\hline Chronic bronchitis & $13(23.6)$ \\
\hline Anemia & $11(20.0)$ \\
\hline Dementia & $13(23.6)$ \\
\hline Cachexia & $1(0.2)$ \\
\hline
\end{tabular}

Values are presented as means \pm SD or numbers (\%) unless otherwise stated.

tancy; (b) medications of uncertain appropriateness, i.e., drugs that need a case-by-case evaluation because they could have a role in end-of-life patients but their real effectiveness is questionable on account of the short life expectancy; and (c) potentially appropriate treatments, i.e., drugs that provide symptomatic relief or that should be tapered slowly over several weeks or months in order to avoid withdrawal symptoms, such as psychotropic drugs or proton pump inhibitors [15]. A geriatrician and a clinical pharmacologist separately analyzed drug prescriptions and resolved any discordances with a final round.

\section{Statistical Analysis}

The patients' sociodemographic characteristics were compared using univariate analysis by $\chi^{2}$ tests for categorical variables and $t$ tests or matched pair $t$ tests for continuous variables. The $\mathrm{McNe}$ mar test was used to compare drug treatments at admission and discharge. $p<0.05$ were considered statistically significant. Analyses were done using JMP Pro 12 (SAS Institute Inc., Cary, USA).

\section{Results}

The sample comprised 55 end-of-life patients recruited among the patients discharged by the internal medicine and geriatric wards of REPOSI. Follow-up data were available for 30 of those discharged in critical conditions; 27 (90\%) died between discharge and the 3-month follow-up, confirming their limited life expectancy. The main sociodemographic characteristics and diagnoses are reported in Table 1. Patients had high rates of comorbidity and most of them suffered from cardiovascular diseases. About $72 \%$ had a caregiver and most had cognitive impairment consistent with a diagnosis of dementia. Half of the patients had been admitted in the previous 6 months and were bedridden at discharge with pressure sores.

The mean number of drugs remained the same from admission to discharge (mean \pm SD: $5.5 \pm 3.6$ and $5.5 \pm$ $3.4 ; p=0.97)$. Table 2 lists the drugs that we considered as potentially avoidable preventive medications in endof-life patients, those of uncertain appropriateness, and those that were potentially appropriate. There were significantly fewer patients with at least 1 preventive medication that could be considered for deprescription at the end of life from admission to discharge $(p=0.02)$. Angiotensin-converting-enzyme inhibitors, angiotensin receptor blockers, calcium channel blockers, lipid-lowering drugs, and clonidine were the most frequent potentially avoidable cardiovascular medications prescribed at discharge, followed by xanthine oxidase inhibitors and drugs to prevent fractures. Drugs for peptic ulcer and gastroesophageal reflux disease, analgesics, psychotropic drugs, and systemic corticosteroids were the potentially appropriate symptomatic medications maintained at discharge.

Patients exposed to at least 1 potentially severe DDI slightly increased between admission $(31 ; 56.4 \%)$ and discharge $(37 ; 67.3 \% ; p=0.18)$; drugs that concomitantly increased the risk of QT prolongation and torsades de pointes, followed by those that increased the risk of bleeding, were the most frequent potentially severe DDI (Table 3).

\section{Discussion}

Identification of the end of life should bring about a significant reduction in the number of daily drugs, but this was not observed in the present study. Hospital discharge was associated with only a small, though significant, reduction in the use of preventive medications that are commonly prescribed. However, these results could be further 
Table 2. Main pharmacological treatment of patients discharged in critical condition

\begin{tabular}{|c|c|c|}
\hline & \multicolumn{2}{|l|}{ Patients } \\
\hline & admission & discharge \\
\hline \multicolumn{3}{|c|}{ Potentially avoidable preventive medications } \\
\hline At least 1 & $30(54.5)$ & $21(38.2)$ \\
\hline \multicolumn{3}{|l|}{ Cardiovascular drugs } \\
\hline Omega-3 & 1 & 1 \\
\hline Statins & 5 & 3 \\
\hline Clonidine & 3 & 3 \\
\hline ACE inhibitors or ARB & 15 & 9 \\
\hline Calcium channel blockers & 10 & 4 \\
\hline \multicolumn{3}{|l|}{ Hematological agents } \\
\hline Iron & - & 1 \\
\hline Folic acid & - & 2 \\
\hline \multicolumn{3}{|l|}{ Antidiabetic agents } \\
\hline Oral antidiabetic & 4 & 0 \\
\hline \multicolumn{3}{|l|}{ Other } \\
\hline Xanthine oxidase inhibitors & 11 & 5 \\
\hline Calcium carbonate & 1 & 1 \\
\hline Cholecalciferol & - & 1 \\
\hline \multicolumn{3}{|c|}{ Preventive medications of uncertain appropriateness } \\
\hline At least 1 & $34(61.8)$ & $30(54.5)$ \\
\hline \multicolumn{3}{|l|}{ Hematological agents } \\
\hline Low-molecular-weight heparin & 12 & 20 \\
\hline Erythropoietin-stimulating agents & 2 & 4 \\
\hline Antiplatelets & 18 & 7 \\
\hline Oral anticoagulants & 4 & 4 \\
\hline \multicolumn{3}{|l|}{ Other } \\
\hline Antineoplastic agents & 1 & 0 \\
\hline Immunosuppressants & 2 & 0 \\
\hline Doxophylline & - & 1 \\
\hline Albumin & 1 & 2 \\
\hline Cholestyramine $^{a}$ & - & 1 \\
\hline
\end{tabular}

improved since in fact about $40 \%$ of patients were discharged with avoidable medications. Cardiovascular drugs were the potentially avoidable preventive medications most frequently prescribed at admission that were partially reduced at discharge. Most cardiovascular drugs usually have no place in end-of-life patients because the time to benefit is clearly shorter than the life expectancy [2]; when prescribed to prevent diabetic nephropathy or reduce mortality from heart failure angiotensin-converting-enzyme inhibitors, angiotensin receptor blockers, and calcium channel blockers (excluding diltiazem or verapamil) are of little value when a patient's life expectancy is severely curtailed as a result of other irreversible disorders. Similarly, lipid-lowering drugs are almost always inappropriate at the end of life. There were definite reductions in the prescription of oral antidiabetics and xanthine oxidase inhibitors at discharge. Stopping these drugs is generally appro-

\begin{tabular}{|c|c|c|}
\hline & \multicolumn{2}{|l|}{ Patients } \\
\hline & admission & discharge \\
\hline \multicolumn{3}{|l|}{ Potentially appropriate drugs } \\
\hline At least 1 & $50(90.9)$ & $47(85.5)$ \\
\hline \multicolumn{3}{|l|}{ Cardiovascular drugs } \\
\hline High-ceiling diuretic & 21 & 19 \\
\hline$\beta$-Blockers & 15 & 10 \\
\hline Diltiazem & 1 & 1 \\
\hline Potassium-sparing diuretics & 9 & 5 \\
\hline Long-acting nitrates ${ }^{\mathrm{b}}$ & 7 & 7 \\
\hline Cardiac therapy & 9 & 8 \\
\hline Analgesics & 11 & 17 \\
\hline \multicolumn{3}{|l|}{ Gastrointestinal drugs } \\
\hline Drug for peptic ulcer and GERD & 35 & 36 \\
\hline Laxatives & 3 & 4 \\
\hline Prokinetics & 2 & 3 \\
\hline \multicolumn{3}{|l|}{ Psychotropic drugs } \\
\hline Antidepressants ${ }^{c}$ & 7 & 5 \\
\hline Hypnotic sedatives & 5 & 3 \\
\hline Antipsychotics & 4 & 5 \\
\hline \multicolumn{3}{|l|}{ Antidiabetic agents } \\
\hline Insulin & 10 & 11 \\
\hline \multicolumn{3}{|l|}{ Other } \\
\hline Systemic corticosteroids & 10 & 20 \\
\hline Antiasthmatics & 11 & 8 \\
\hline Antibiotics & 8 & 16 \\
\hline Alpha antagonists for $\mathrm{BPH}$ & 5 & 4 \\
\hline \multicolumn{3}{|l|}{ Testosterone $5 a$-reductase } \\
\hline inhibitors & 2 & 2 \\
\hline Antiepileptics & 9 & 7 \\
\hline Antifungals & 1 & 4 \\
\hline Antiparkinson & 3 & 1 \\
\hline Thyroid therapy & 6 & 5 \\
\hline
\end{tabular}

Values are presented as numbers (\%) or numbers. ACE, angiotensin-converting-enzyme; $\mathrm{ARB}$, angiotensin II receptor antagonist; $\mathrm{BPH}$, benign prostatic hypertrophy; GERD, gastroesophageal reflux disease. ${ }^{a}$ To relieve itching caused by partial biliary obstruction. ${ }^{b}$ Including nitroglycerin and isosorbide. ${ }^{\mathrm{c}}$ Including trazodone, venlafaxine, and duloxetine; $p=0.02$.

priate because the goals for managing diabetes change at the end of life since it is no longer important to prevent the long-term effects of hyperglycemia. Similarly, treating asymptomatic hyperuricemia to prevent gout is clearly inappropriate [16] in the end-of-life patient.

Among the preventive medications of uncertain appropriateness, the use of low-molecular-weight heparin increased at discharge while the proportion of patients treated with antiplatelet drugs decreased; these drugs could be useful in preventing thrombotic events, although they increase the risk of bleeding in older and frailer pa- 
Table 3. Most frequent potentially severe DDI

\begin{tabular}{|c|c|c|c|}
\hline Anticoagulants or antiplatelets + antiplatelets or SSRI & Increased risk of bleeding & $10(18.2)$ & $11(20.0)$ \\
\hline Digoxin + high-ceiling diuretic & Increased risk of digoxin toxicity & $4(7.3)$ & $5(9.1)$ \\
\hline Statin + inhibitor of hepatic metabolism ${ }^{\mathrm{a}}$ & $\begin{array}{l}\text { Increased risk of myopathy including } \\
\text { rhabdomyolysis }\end{array}$ & $2(3.6)$ & $2(3.6)$ \\
\hline Fluoroquinolones + systemic corticosteroid & Increased risk of tendon rupture & $2(3.6)$ & $1(1.8)$ \\
\hline
\end{tabular}

Values are presented as numbers (\%). SSRI, selective serotonin reuptake inhibitors; ACE, angiotensin converting enzyme. a Ticagrelor.

tients who often fall. It is hard to assess the appropriateness of these medications and a case-by-case evaluation is needed, although anticoagulants and antiplatelets were frequently involved in potentially severe DDI at discharge, increasing the risk of adverse drug events. Because deprescribing must consider not only the risks of individual drugs but also the cumulative risk of DDI [17], the higher percentage of patients exposed to potentially severe DDI emphasizes the need for closer evaluation of drugs prescribed to end-of-life patients.

Our findings suggest that the review of drug medications, optimizing polypharmacy and deprescribing could be further improved in older adults discharged with a very limited life expectancy (e.g., less than 3 months). A consensus framework, or shared criteria with deprescribing guidelines for potentially inappropriate medication, could be useful in rationalizing drug therapy. Careful consideration of a patient's life expectancy, the time to benefit of treatments, goals of care, and treatment targets for each drug (including those used for a long time before the end-of-life period) is important for recommending any rational framework for decision making $[2,5,18]$.

There may be several reasons for physicians not considering the discontinuation of futile medications in patients with a limited life expectancy. Scant awareness, as well the reactions of patients or their relatives, seems to be an important factor [19]. Unexpectedly, patients may be more willing to stop unnecessary medications than their physicians believe, as observed in a study of older patients with multiple chronic morbidities who were favorable to discontinue medication in $90 \%$ of cases [20].
Emphasizing the positive aspects of stopping medicines, such as reducing the burden of taking pills, rather than insisting on the uselessness of continuation, may be a helpful approach [21] that physicians could emphasize in conversations with patients and relatives.

The strength of this study is that this it analyzes how the hospital transition can be associated with changes in preventive and symptomatic medications by internal medicine and geriatric specialists for patients with a limited life expectancy.

This study has some limitations. First, REPOSI was not specifically designed to collect information about patients with a limited life expectancy and only those able to give written informed consent were enrolled, thus excluding those who were too frail to give consent. The sample of patients is very small, because we focused on patients in 'critical conditions', so we could assess the use of preventive or symptomatic drugs in patients with a limited life expectancy according to the clinical evaluation.

\section{Conclusion}

Hospital discharge is associated with a small reduction in the use of commonly prescribed preventive medications in patients discharged with a limited life expectancy. Cardiovascular drugs are the most frequent potentially avoidable preventive medications. A consensus framework or shared criteria for potentially inappropriate medication in elderly patients with a limited life expectancy could help improve drug prescription. 


\section{Acknowledgement}

REPOSI is a network of Italian internal medicine hospital wards which, voluntarily and with no financial support, agreed to participate in the collection of data for this study over the 4 index weeks (see the Appendix for a list of investigators and coauthors of the REPOSI Study Group). The authors are grateful to J.D. Baggott for editorial assistance.

\section{Disclosure Statement}

The authors declare no conflict of interests.

\section{Appendix}

The investigators and coauthors of the REPOSI Study Group are as follows:

Steering committee: Pier Mannuccio Mannucci (Chair, Fondazione IRCCS Cà Granda Ospedale Maggiore Policlinico, Milan, Italy); Alessandro Nobili (Cochair, IRCCS-Istituto di Ricerche Farmacologiche Mario Negri, Milan, Italy); Mauro Tettamanti, Luca Pasina, and Carlotta Franchi (IRCCS-Istituto di Ricerche Farmacologiche Mario Negri); Salvatore Corrao (ARNAS Civico, Di Cristina, Benfratelli, DiBiMIS, Università di Palermo, Palermo, Italy); Alessandra Marengoni (Spedali Civili di Brescia, Brescia, Italy); Francesco Salerno (IRCCS Policlinico San Donato Milanese, Milan, Italy), Matteo Cesari (UO Geriatria, Università degli Studi di Milano), Francesco Perticone (President, SIMI), Giuseppe Licata (Medicina Interna e Cardioangiologia, Azienda Ospedaliera Universitaria Policlinico P. Giaccone di Palermo, Palermo, Italy); Francesco Violi (Prima Clinica Medica, Policlinico Umberto I, Rome, Italy); and Gino Roberto Corazza, (Clinica Medica I, Reparto 11, IRCCS Policlinico San Matteo di Pavia, Pavia, Italy).

Clincal data monitoring and revision: Carlotta Franchi and Laura Cortesi (IRCCS-Istituto di Ricerche Farmacologiche Mario Negri).

Database management and statistics: Mauro Tettamanti, Laura Cortesi, and Ilaria Ardoino (IRCCS-Istituto di Ricerche Farmacologiche Mario Negri).

\section{Investigators}

Italian Hospitals

Domenico Prisco, Elena Silvestri, Caterina Cenci, and Giacomo Emmi (Medicina Interna Interdisciplinare, Azienda Ospedaliero Universitaria Careggi Firenze); Gianni Biolo, Michela Zanetti, Martina Guadagni, and Michele Zaccari (Clinica Medica Generale e Terapia Medica, Azienda Sanitaria Universitaria Integrata di Trieste); Massimo Vanoli, Giulia Grignani, and Edoardo Alessandro Pulixi (Medicina Interna, Azienda Ospedaliera della Provincia di Lecco, Ospedale di Merate, Lecco); Mauro Bernardi, Silvia Li Bassi, Luca Santi, and Giacomo Zaccherini (Semeiotica Medica Bernardi, Azienda Ospedaliera Policlinico Sant'Orsola-Malpighi, Bologna); Elmo Mannarino, Graziana Lupattelli, Vanessa Bianconi, and Francesco Paciullo (Medicina Interna, Azienda Ospedaliera Santa Maria della Misericordia, Perugia); Ranuccio Nuti, Roberto Valenti, Martina Ruvio, Silvia Cappelli, and Alberto Palazzuoli (Medicina Interna I, Azienda Ospedaliera Università Senese, Siena);
Oliviero Olivieri, Domenico Girelli, and Thomas Matteazzi (Medicina Generale a indirizzo Immuno-Ematologico e Emocoagulativo, Azienda Ospedaliera Universitaria Integrata di Verona, Verona); Mario Barbagallo, Ligia Dominguez, Floriana Cocita, Vincenza Beneduce, and Lidia Plances (Unità Operativa di Geriatria e Lungodegenza, Azienda Ospedaliera Universitaria Policlinico P. Giaccone di Palermo); Marco Zoli, Ilaria Lazzari, and Mattia Brunori (Unità Operativa di Medicina Interna, Azienda Ospedaliera Universitaria Policlinico Sant'Orsola-Malpighi); Franco Laghi Pasini and Pier Leopoldo Capecchi (Unità Operativa Complessa Medicina 2, Azienda Ospedaliera Università Senese); Giuseppe Palasciano, Maria Ester Modeo, and Carla Di Gennaro (Medicina Interna Ospedaliera L. D’Agostino, Medicina Interna Universitaria A. Murri, Azienda Ospedaliero-Universitaria Consorziale Policlinico di Bari, Bari); Maria Domenica Cappellini, Diletta Maira, Valeria Di Stefano, Giovanna Fabio, Sonia Seghezzi, and Marta Mancarella (Unità Operativa Medicina Interna IA, Fondazione IRCCS Cà Granda Ospedale Maggiore Policlinico); Matteo Cesari, Paolo Dionigi Rossi, Sarah Damanti, Marta Clerici, and Federica Conti (Geriatria, Fondazione IRCCS Cà Granda Ospedale Maggiore Policlinico); Gino Roberto Corazza, Emanuela Miceli, Marco Vincenzo Lenti, Martina Pisati, and Costanza Caccia Dominioni (Clinica Medica I, Reparto 11, IRCCS Policlinico San Matteo di Pavia); Giovanni Murialdo, Alessio Marra, and Federico Cattaneo (IRCS Azienda Ospedaliera Universitaria San Martino-IST di Genova, Genoa); Maria Beatrice Secchi and Davide Ghelfi (Divisione Medicina, Ospedale Bassini di Cinisello Balsamo, Milan); Luigi Anastasio, Lucia Sofia, and Maria Carbone (Medicina Interna, Ospedale Civile Jazzolino di Vibo Valentia, Vibo Valentia); Francesco Cipollone, Maria Teresa Guagnano, Ermanno Angelucci, and Emanuele Valeriani (Clinica Medica, Ospedale Clinicizzato SS. Annunziata, Chieti); Gerardo Mancuso, Daniela Calipari, and Mosè Bartone (Unità Operativa Complessa Medicina Interna, Ospedale Giovanni Paolo II Lamezia Terme, Catanzaro); Giuseppe Delitala and Maria Berria (Clinica Medica, Azienda Ospedaliera-Universitaria di Sassari); Maurizio Muscaritoli, Alessio Molfino, and Enrico Petrillo (Medicina Interna e Nutrizione Clinica, Policlinico Umberto I, Sapienza Università di Roma); Giuseppe Zuccalà and Gabriella D'Aurizio (Unità Operativa Complessa Medicina d'Urgenza e Pronto Soccorso, Policlinico Universitario A. Gemelli, Rome); Giuseppe Romanelli, Alessandra Marengoni, and Alberto Zucchelli (Geriatria, Spedali Civili di Brescia); Antonio Picardi, Umberto Vespasiani Gentilucci, Paolo Gallo, and Chiara Dell'Unto (Medicina Clinica-Epatologia, Università Campus Biomedico, Rome); Giorgio Annoni, Maurizio Corsi, Giuseppe Bellelli, Sara Zazzetta, Paolo Mazzola, Hajnalka Szabo, and Alessandra Bonfanti (Unità Operativa di Geriatria, Università degli studi di Milano-Bicocca Ospedale S. Gerardo, Monza); Franco Arturi, Elena Succurro, and Mariangela Rubino (Unità Operativa Complessa di Medicina Interna, Università degli Studi Magna Grecia, Policlinico Mater Domini, Catanzaro); Maria Grazia Serra and Maria Antonietta Bleve (Unità Operativa Complessa Medicina, Azienda Ospedaliera Cardinale Panico Tricase, Lecce); Laura Gasbarrone and Maria Rosaria Sajeva (Medicina Interna 1, Azienda Ospedaliera Ospedale San Camillo Forlanini, Rome); Antonio Brucato and Silvia Ghidoni (Medicina 1, Azienda Ospedaliera Papa Giovanni XXIII, Bergamo); Fabrizio Fabris, Irene Bertozzi, Giulia Bogoni, Maria Victoria Rabuini, and Elisabetta Cosi (Clinica Medica I, Azienda Ospedaliera Università di Padova, Padua); Roberto Manfredini, Fabio Fabbian, Benedetta Boari, Alfredo De Giorgi, and Ruana Tiseo 
(Unità Operativa Clinica Medica, Azienda Ospedaliera - Universitaria Sant'Anna, Ferrara); Giuseppe Paolisso and Maria Rosaria Rizzo, (VI Divisione di Medicina Interna e Malattie Nutrizionali dell'Invecchiamento, Azienda Ospedaliera Universitaria della Seconda Università degli Studi di Napoli, Naples); Claudio Borghi, Enrico Strocchi, Valeria De Sando, and Ilenia Pareo (Unità Operativa di Medicina Interna Borghi, Azienda Ospedaliera Universitaria Policlinico Sant'Orsola-Malpighi); Carlo Sabbà, Francesco Saverio Vella, Patrizia Suppressa, Pasquale Agosti, Andrea Schilardi, and Francesca Loparco (Medicina Interna Universitaria C. Frugoni, Azienda Ospedaliero-Universitaria Consorziale Policlinico di Bari); Luigi Fenoglio, Christian Bracco, and Alessia Valentina Giraudo (S.C. Medicina Interna, Azienda Sanitaria Ospedaliera Santa Croce e Carle di Cuneo, Cuneo); Silvia Fargion, Giulia Periti, Marianna Porzio, and Slivia Tiraboschi (Medicina Interna 1B, Fondazione IRCCS Cà Granda Ospedale Maggiore Policlinico); Flora Peyvandi, Raffaella Rossio, Barbara Ferrari, and Giulia Colombo (Medicina Interna 2, Fondazione IRCCS Cà Granda Ospedale Maggiore Policlinico); Valter Monzani, Valeria Savojardo, Christian Folli and Giuliana Ceriani (Medicina Interna Alta Intensità, Fondazione IRCCS Cà Granda Ospedale Maggiore Policlinico); Francesco Salerno and Giada Pallini (Medicina Interna, IRCCS Policlinico San Donato e Università di Milano, San Donato Milanese); Franco Dallegri, Luciano Ottonello, Luca Liberale, Lara Caserza and Kassem Salam (Medicina Interna 1, Università di Genova, Genoa); Nicola Lucio Liberato and Tiziana Tognin (ASST di Pavia, UOSD Medicina Interna, Ospedale di Casorate Primo, Pavia); Giovanni Battista Bianchi and Sabrina Giaquinto (Ospedale SS Gerosa e Capitanio di Lovere, Bergamo, and Unità Operativa Complessa di Medicina Generale, Azienda Ospedaliera Bolognini di Seriate, Bergamo); Francesco Purrello, Antonino Di Pino, and Salvatore Piro (Unità Operativa Complessa di Medicina Interna, Ospedale Garibaldi Nesima, Catania); Renzo Rozzini, Lina Falanga, Elena Spazzini, and Camillo Ferrandina (Medicina Interna e Geriatria, Ospedale Poliambulanza, Brescia); Giuseppe Montrucchio and Paolo Petitti (Medicina Interna 2 U. Indirizzo d'Urgenza, Dipartimento di Scienze Mediche, Università di Torino, Città della Scienza e della Salute, Torino); Raffaella Salmi and Piergiorgio Gaudenzi (Unità Operativa di Medicina Ospedaliera II, Azienda Ospedaliera-Universitaria Sant'Anna); Francesco Violi and Ludovica Perri (Prima Clinica Medica, Policlinico Umberto I); Raffaele Landolfi, Massimo Montalto, and Antonio Mirijello (Clinica Medica, Policlinico Universitario A. Gemelli); Luigina Guasti, Luana Castiglioni, Andrea Maresca, Alessandro Squizzato, Marta Molaro, and Alessandra Grossi (Medicina Interna I, Università degli Studi dell'Insubria, Ospedale di Circolo e Fondazione Macchi, Varese); Marco Bertolotti, Chiara Mussi, Maria Vittoria Libbra, Giulia Dondi, Elisa Pellegrini, and Lucia Carulli (Unità Operativa di Geriatria e U.O. di Medicina a indirizzo Metabolico Nutrizionistico, Nuovo Ospedale Civile, Università di Modena e Reggio Emilia, AUSL di Modena, Modena,); Francesco Perticone, Lidia Colangelo, and Tania Falbo (Unità Operativa Malattie Cardiovascolari Geriatriche, Università Magna Grecia, Policlinico Mater Domini); Vincenzo Stanghellini, Roberto De Giorgio, Eugenio Ruggeri and Sara del Vecchio (Dipartimento di Scienze Mediche e Chirurgiche, Unità Operativa di Medicina Interna, Università degli Studi di Bologna/ Azienda Ospedaliero-Universitaria Sant'Orsola-Malpighi); Andrea Salvi, Roberto Leonardi, and Giampaolo Damiani (U.O. 3a Medicina Generale, Spedali Civili di Brescia); Armando Gabrielli, William Capeci, Massimo Mattioli, Giuseppe Pio Martino, Lorenzo
Biondi, and Pietro Pettinari (Clinica Medica, Azienda Ospedaliera Universitaria - Ospedali Riuniti di Ancona); Riccardo Ghio and Anna Dal Col (Medicina III, Azienda Ospedaliera Università San Martino); Salvatore Minisola and Luciano Colangelo (Medicina Interna F e Malattie Metaboliche dell'osso, Policlinico Umberto I); Antonella Afeltra, Benedetta Marigliano, and Maria Elena Pipita (Medicina Clinica, Policlinico Campus Biomedico Roma, Rome); Pietro Castellino, Julien Blanco, Luca Zanoli, and Samuele Pignataro (Dipartimento di Medicina, Azienda Ospedaliera Universitaria Policlinico - V. Emanuele, Catania); Valter Saracco, Marisa Fogliati, and Carlo Bussolino (Medicina A, Ospedale Cardinal Massaia Asti); Francesca Mete and Miriam Gino (Medicina Interna, Ospedale degli Infermi di Rivoli, Torino); Antonio Cittadini, Carlo Vigorito, Michele Arcopinto, Andrea Salzano, Emanuele Bobbio, Alberto Maria Marra, and Domenico Sirico (Medicina Interna e Riabilitazione Cardiologica, Azienda Policlinico Universitario Federico II di Napoli, Naples); Guido Moreo, Francesca Gasparini, Silvia Prolo, and Gloria Pina (Unità Operativa di Medicina Interna, Clinica San Carlo Casa di Cura Polispecialistica, Paderno Dugnano, Milan); Alberto Ballestrero and Fabio Ferrando (Clinica Di Medicina Interna ad Indirizzo Oncologico, Azienda Ospedaliera Università San Martino di Genova); Sergio Berra, Simonetta Dassi, and Maria Cristina Nava (Medicina Interna, Azienda Ospedaliera Guido Salvini, Garnagnate, Milan); Bruno Graziella, Stefano Baldassarre, Salvatore Fragapani, and Gabriella Gruden (Medicina Interna III, Ospedale S. Giovanni Battista Molinette, Torino); Giorgio Galanti, Gabriele Mascherini, Cristian Petri, and Laura Stefani (Agenzia di Medicina dello Sport, AOUC Careggi, Florence); Margherita Girino and Valeria Piccinelli (Medicina Interna, Ospedale S. Spirito Casale Monferrato, Alessandria); Francesco Nasso, Vincenza Gioffrè, and Maria Pasquale (Struttura Operativa Complessa di Medicina Interna, Ospedale Santa Maria degli Ungheresi, Reggio Calabria); Giuseppe Scattolin, Sergio Martinelli, and Mauro Turrin (Medicina Interna, Ospedale di Monselice, $\mathrm{Pa}$ dua); Leonardo Sechi, Cristina Catena, and Gianluca Colussi (Clinica Medica, Azienda Ospedaliera Universitaria, Udine); Nicola Passariello, Luca Rinaldi (Medicina Interna, Presidio Medico di Marcianise, Naples); Franco Berti, Giuseppe Famularo, and Tarsitani Patrizia (Medicina Interna II, Azienda Ospedaliera San Camillo Forlanini); Roberto Castello and Michela Pasino (Medicina Generale e Sezione di Decisione Clinica, Ospedale Civile Maggiore Borgo Trento, Verona); Gian Paolo Ceda, Marcello Giuseppe Maggio, Simonetta Morganti, and Andrea Artoni (U.O.C Clinica Geriatrica, Azienda Ospedaliero Universitaria di Parma); Stefano Del Giacco, Davide Firinu, Francesca Losa, and Giovanni Paoletti (Medicina Interna, Allergologia ed Immunologia Clinica, Policlinico Universitario Dulio Casula, Azienda Ospedaliero-Universitaria di Cagliari, Cagliari); Giuseppe Montalto, Anna Licata, and Valentina Malerba (U.O.S Prevenzione Malattie Epatobiliari, Azienda Ospedaliera Universitaria Policlinico P. Giaccone di Palermo); Lasco Antonino, Giorgio Basile, and Catalano Antonino (Unità Operativa di Geriatria, Azienda Ospedaliera Universitaria Policlinico G. Martino, Messina); Lorenzo Malatino, Benedetta Stancanelli, Valentina Terranova, and Salvatore Di Marca (Azienda Ospedaliera per l'Emergenza Cannizzaro andClinica Medica Università di Catania, Catania); Patrizia Mecocci, Carmelinda Ruggiero, and Virginia Boccardi (Struttura Complessa di Geriatria, Università degli Studi di Perugia-Azienda Ospedaliera Santa Maria della Misericordia); Tiziana Meschi, Fulvio Lauretani, and Andrea Ticinesi (U.O. Medicina Interna e Lungodegenza Critica, Azienda Ospedaliera 
Universitaria di Parma); Pietro Minuz and Luigi Fondrieschi (Medicina Generale per lo Studio ed il Trattamento dell'Ipertensione Arteriosa, Azienda Ospedaliera Universitaria Verona, Policlinico GB Rossi, Verona); Mario Pirisi, Gian Paolo Fra, and Daniele Sola (Medicina Interna 1, Azienda Ospedaliera Universitaria Maggiore della Carità); Massimo Porta and Piero Riva (Medicina Interna 1U, Azienda Ospedaliera Universitaria Città della Salute e della Scienza di Torino); Roberto Quadri (S.C. Medicina Interna, Ospedale di Ciriè, ASL TO4, Torino); Giorgio Scanzi, Caterina Mengoli, Stella Provini, and Laura Ricevuti (Medicina, ASST Lodi, Presidio di Codogno, Milan); Emilio Simeone, Rosa Scurti, and Fabio Tolloso (Geriatria, Ospedale Spirito Santo di Pescara); Roberto Tarquini, Alice Valoriani, Silvia Dolenti, and Giulia Vannini (Medicina Interna I, Ospedale San Giuseppe, Empoli, USL Toscana Centro, Florence); Alberto Tedeschi and Lucia Trotta (Medicina Interna a indirizzo Pneumologico, ASST Fatebenefratelli - Sacco, Milan); Riccardo Volpi, Pietro Bocchi, and Alessandro Vignali (Clinica e Terapia Medica, Azienda Ospedaliera Universitaria di Parma); and Sergio Harari, Chiara Lonati, and Mara Cattaneo (U.O. Medicina Generale, Ospedale San Giuseppe Multimedica Spa)

\section{Spanish Hospitals}

Nieves Ramirez Duque (Hospital Universitario Virgen del Rocio, Sevilla); Alberto Muela Molinero (Hospital de Leon); Pedro Abad Requejo, Vanessa Lopez Pelaez, and Lara Tamargo (Hospital del Oriente de Asturias, Arriondas); Xavier Corbella Viros and Francesc Formiga (Hospital Universitario de Bellvitge); Jesus Diez Manglano, Esperanza Bejarano Tello, Esther Del Corral Behamonte, and Maria Sevil Puras (Hospital Royo Villanova, Zaragoza); Manuel Romero (Hospital Infanta Elena Huelva); Blanca Pinilla Llorente, Cristina Lopez Gonzalez-Cobos, and M. Victoria Villalba Garcia (Hospital Gregorio Marañon, Madrid); Saez Lopez and Juan Bosco (Hospital Universitario de Puerto Real, Cadiz); Susana Sanz Baena, Marta Arroyo Gallego (Hospital Del Henares De Coslada, Madrid); Concepcion Gonzalez Becerra, Antonio Fernandez Moyano, Mercedes Gomez Hernandez, and Manuel Poyato Borrego (Hospital San Juan De Dios Del Aljarafe, Sevilla); Raquel Pacheco Cuadros, Florencia Perez Rojas, Beatriz Garcia Olid, and Sara Carrascosa Garcia (Hospital Virgen De La Torre De Madrid); Alfonso Gonzalez-Cruz Cervellera, Marta Peinado Martinez, and Sara Carrascosa Garcia (Hospital General Universitario De Valencia); Alberto Ruiz Cantero, Antonio Albarracín Arraigosa, Montserrat Godoy Guerrero, and Miguel Ángel Barón Ramos (Hospital De La Serrania De Ronda); Machin Jose Manuel (Hospital Universitario De Guadalajara); Ignacio Novo Veleiro, Lucía Alvela Suarez (Hospital Universitario De Santiago De Compostela); Alfonso Lopez, David Rubal Bran, and Iria Iñiguez Vazquez (Hospital Lucus Augusti De Lugo); and Monica Rios Prego (Hospital Universitario De Pontevedra).

\section{References}

1 O'Mahony D, O'Connor MN. Pharmacotherapy at the end-of-life. Age Ageing. $2011 \mathrm{Jul}$; 40(4):419-22.

2 Cruz-Jentoft AJ, Boland B, Rexach L. Drug therapy optimization at the end of life. Drugs Aging. 2012 Jun;29(6):511-21.

3 Blass DM, Black BS, Phillips H, Finucane T, Baker A, Loreck D, et al. Medication use in nursing home residents with advanced dementia. Int J Geriatr Psychiatry. 2008 May; 23(5):490-6.

4 Riechelmann RP, Krzyzanowska MK, Zimmermann C. Futile medication use in terminally ill cancer patients. Support Care Cancer. 2009 Jun;17(6):745-8.

5 Holmes HM, Hayley DC, Alexander GC, Sachs GA. Reconsidering medication appropriateness for patients late in life. Arch Intern Med. 2006 Mar;166(6):605-9.

6 Fried TR, O'Leary J, Towle V, Goldstein MK, Trentalange M, Martin DK. Health outcomes associated with polypharmacy in community-dwelling older adults: a systematic review. J Am Geriatr Soc. 2014 Dec; 62(12):2261-72.

7 Spinewine A, Schmader KE, Barber N, Hughes C, Lapane KL, Swine C, et al. Appropriate prescribing in elderly people: how well can it be measured and optimised? Lancet. 2007 Jul;370(9582):173-84.

8 Hajjar ER, Cafiero AC, Hanlon JT. Polypharmacy in elderly patients. Am J Geriatr Pharmacother. 2007 Dec;5(4):345-51.
9 Morgan NA, Rowett D, Currow DC. Analysis of drug interactions at the end of life. BMJ Support Palliat Care. 2015 Sep;5(3):281-6.

10 Frechen S, Zoeller A, Ruberg K, Voltz R, Gaertner J. Drug interactions in dying patients: a retrospective analysis of hospice inpatients in Germany. Drug Saf. 2012 Sep; 35(9):745-58.

11 Maddison AR, Fisher J, Johnston G. Preventive medication use among persons with limited life expectancy. Prog Palliat Care. 2011 Jan;19(1):15-21.

12 Wright JM, Musini VM. First-line drugs for hypertension. Cochrane Database Syst Rev. 2009 Jul;(3):CD001841.

13 Kashani A, Phillips CO, Foody JM, Wang Y, Mangalmurti S, Ko DT, et al. Risks associated with statin therapy: a systematic overview of randomized clinical trials. Circulation. 2006 Dec;114(25):2788-97.

14 Reid DM, Hosking D, Kendler D, Brandi ML, Wark JD, Marques-Neto JF, et al. A comparison of the effect of alendronate and risedronate on bone mineral density in postmenopausal women with osteoporosis: 24-month results from FACTS-International. Int J Clin Pract. 2008 Apr;62(4):575-84.

15 A practical guide to stopping medicine in older people. Best Pract J. 2010;27:11-22.

16 Pasina L, Brucato AL, Djade CD, Di Corato P, Ghidoni S, Tettamanti M, et al.; REPOSI Investigators. Inappropriate prescription of allopurinol and febuxostat and risk of adverse events in the elderly: results from the REPOSI registry. Eur J Clin Pharmacol. 2014 Dec; 70(12):1495-503

17 Scott IA, Hilmer SN, Reeve E, Potter K, Le Couteur D, Rigby D, et al. Reducing inappropriate polypharmacy: the process of deprescribing. JAMA Intern Med. 2015 May; 175(5):827-34

18 Steinman MA, Hanlon JT. Managing medications in clinically complex elders: "There's got to be a happy medium". JAMA. 2010 Oct; 304(14):1592-601.

19 Geijteman EC, Dees MK, Tempelman MM, Huisman BA, Arevalo JJ, Perez RS, et al. Understanding the Continuation of Potentially Inappropriate Medications at the End of Life: Perspectives from Individuals and Their Relatives and Physicians. J Am Geriatr Soc. 2016 Dec;64(12):2602-4.

20 Reeve E, Wiese MD, Hendrix I, Roberts MS, Shakib S. People's attitudes, beliefs, and experiences regarding polypharmacy and willingness to Deprescribe. J Am Geriatr Soc. 2013 Sep;61(9):1508-14

21 Reeve E, Shakib S, Hendrix I, Roberts MS, Wiese MD. Review of deprescribing processes and development of an evidence-based, patient-centred deprescribing process. Br J Clin Pharmacol. 2014 Oct;78(4):738-47.

22 Crediblemeds [Internet]. Risk categories for drugs that prolong QT \& induce torsades de pointes (TdP) [cited 2018 Jan 10]. Available from: https://www.crediblemeds.org. 